\title{
Nickel(II) 3,4;9,10-Perylenediimide bis-Phosphonate Pentahydrate: A Metal-Organic Ferromagnetic Dye
}

\author{
Carlo Bellitto, $^{\dagger}$ Guido Righini, ${ }^{\dagger}$ Carlos J. Gómez-García, ${ }^{\S}$ Ruggero Caminiti, ${ }^{\|}$Marilena Carbone, ${ }^{\perp}$ \\ Roberto Matassa, ${ }^{*}, *$ and Elvira M. Bauer ${ }^{*}, \dagger$ \\ ${ }^{\dagger}$ Istituto di Struttura della Materia, CNR, Via Salaria km 29.300, 00015 Monterotondo (Italy) \\ "Dipartimento di Scienze di Base e Applicate per l'Ingegneria, Università "Sapienza”, Via A. Scarpa 16, 00161, Roma, (Italy) \\ ${ }^{\S}$ Instituto de Ciencia Molecular, Universidad de Valencia, C/Catedrático José Beltrán 2, 46980 Paterna (Spain) \\ "Dipartimento di Chimica, Università "Sapienza", P.le A. Moro 5, 00185 Roma (Italy) \\ ${ }^{\perp}$ Dipartimento di Scienze e Technologie Chimiche, Università Tor Vergata, Via della Ricerca Scientifica 1, 00133 Roma (Italy)
}

Supporting Information

ABSTRACT: The new metal-organic compound nickel(II) 3,4;9,10perylenediimide bis-phosphonate pentahydrate, i.e. $\mathrm{Ni}_{2}$ [(PDI-BP)$\left.\left(\mathrm{H}_{2} \mathrm{O}\right)_{2}\right] \cdot 3 \mathrm{H}_{2} \mathrm{O}(\mathbf{1})$, has been synthesized and its structural and magnetic properties have been studied. Reaction of 3,4;9,10-perylenediimide bisphosphonate (PDI-BP, hereafter) ligand and nickel chloride in water resulted in the precipitation of a red and poorly crystalline solid (1). As the solid shows a poor crystalline organization of aggregates, the energy dispersive X-ray diffraction analysis (EDXD) technique has been used to obtain short-range order structural information of the single nanoaggregates by radial distribution function analysis. The overall structure of the compound is characterized by layers containing perylene planes shifted in the direction perpendicular to the stacking axes in such a way that only the outer rings overlap. The edges of the perylene planes are connected to the phosphonate groups through an imido group. The oxygen atoms of the $\left[-\mathrm{PO}_{3}\right]^{2-}$ group and those of the water molecules are bonded to the nickel ions resulting in a $\left[\mathrm{NiO}_{6}\right]$ octahedral coordination sphere. The $\mathrm{Ni}-\mathrm{O}$ bond lengths are 0.21 $\pm 0.08 \mathrm{~nm}$ and the $\mathrm{Ni}-\mathrm{O}-\mathrm{Ni}$ angles of aligned moieties are $95 \pm 2^{\circ}$. The oxygen atoms of the water molecules and the nickel atoms are nearly planar and almost perpendicular to the perylene planes forming chains of edge-sharing octahedra. The magnetic properties of (1) show the presence of intrachain ferromagnetic $\mathrm{Ni}-\mathrm{Ni}$ interactions and a long-range ferromagnetic order below $21 \mathrm{~K}$ with a canting angle and with a spin glasslike behavior due to disorder in the inorganic layer. Hysteresis cycles show a coercive field of ca. $272 \mathrm{mT}$ at $2 \mathrm{~K}$ that decreases as the temperature is increased and vanishes at ca. $20 \mathrm{~K}$.

\section{INTRODUCTION}

The design and synthesis of multiproperty materials, such as multiferroics, ${ }^{1}$ ferromagnetic conductors, ${ }^{2}$ porous magnetic MOF solids, ${ }^{3}$ and paramagnetic superconductors ${ }^{4}$ is at the fore-front of solid-state science. The so-called organicinorganic hybrid compounds consisting of organic and inorganic components represent a promising class of materials. ${ }^{5}$ In this context, we use here a strategy consisting in selfassembling a transition metal salt with a suitable functional molecular brick, such as a phosphonate $\mathrm{RPO}_{3} \mathrm{H}_{2}$, or bisphosphonate $\mathrm{H}_{2} \mathrm{O}_{3} \mathrm{P}-\mathrm{R}-\mathrm{PO}_{3} \mathrm{H}_{2}$ moiety $(\mathrm{R}=$ organic group) to prepare metal organo-phosphonates that can be formulated as $\mathrm{M}_{x}\left(\mathrm{RPO}_{3}\right)_{y}(\mathrm{M}=\text { transition metal ion })^{6}{ }^{6}$ This strategy allows the introduction of an additional tailored chemical or physical property in the $\mathrm{R}$ group. In the present work, we have chosen to couple the optical properties of an aromatic molecule as the perylenediimide (PDI) with long-range magnetic ordering provided by properly designed nickel phosphonate.
Aromatic molecules such as 1,4;5,8-naphthalenediimide $(\mathrm{NDI})^{7}$ and 3,$4 ; 9,10$-perylenediimide $(\mathrm{PDI})^{8}$ show high thermal stability, well-defined redox chemistry, and remarkable optical properties. Among them, several perylenediimide derivatives have received considerable attention as dyes and pigments, ${ }^{9}$ as well as electrical conducting materials, ${ }^{10} n$-type semiconductors, ${ }^{11}$ and electroluminescent solids. ${ }^{12}$ The latter properties suggest applications in the field of electronic materials and in the preparation of organic field effect transistors. ${ }^{13,14}$ Moreover, PDI derivatives can easily be reduced in two steps, generating first a radical-anion, followed by a further reduction to a diamagnetic dianion. ${ }^{10}$ Chemical insertion on the PDI molecule of donor groups through the diimide nitrogen atoms or via substitution on the aromatic core provides derivatives whose absorption or emission may be modulated and can be used in the construction of hybrid

Received: April 5, 2012

Published: June 15, 2012 
organic-inorganic lattices. An interesting chemical modification is the insertion of one or two phosphonate groups because they have a well established bonding ability toward metal ions. Thus, phosphonate-substituted aromatic diimides are suitable molecules for the design of multifunctional organic-inorganic hybrids, either as bulk or as thin film, by chemical selfassembling, when left to react with metal ions in water. ${ }^{15}$ Recently, we have reported the synthesis and characterization of a new coordination $\mathrm{Ni}^{\mathrm{II}}$ polymer with 1,$4 ; 5,8$ naphthalene diimide bis-phosphonate, which is a canted antiferromagnet at low temperatures. ${ }^{16}$ However, the poor crystallinity of the latter has precluded the study of its crystal and molecular structures and their correlation with the magnetic properties.

In order to prepare and study new multifunctional ferromagnetic solids, we have synthesized the bis-phosphonate derivative of the aromatic dye 3,4;9,10-Perylenediimide (Chart 1 ), which is characterized by an even more extended $\pi$-electron system ${ }^{9}$ and we have combined this ligand with $\mathrm{Ni}^{\mathrm{II}}$ ions.

Chart 1. Molecular Structure of 3,4;9,10-Perylenediimide bis-Phosphonic Acid (Hereafter PDI-BP)

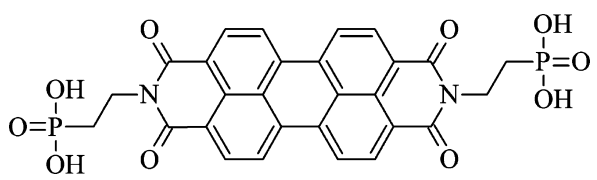

\section{EXPERIMENTAL SECTION}

See the Supporting Information for general information on spectroscopy (FTIR, UV-vis), magnetic and TEM measurements and for energy dispersive X-ray diffraction technique.

General Methods. 3,4;9,10 Perylenetetracarboxylic dianhydride, 2-aminoethylphosphonic acid, $\mathrm{NH}_{2}-\left(\mathrm{CH}_{2}\right)_{2}-\mathrm{PO}_{3} \mathrm{H}_{2}$, and $\left[\mathrm{NiCl}_{2}\right] \cdot 6 \mathrm{H}_{2} \mathrm{O}$ were of analytical grade (Aldrich Chemical Co.) and were used without further purification. Imidazole was purchased from Aldrich and urea was of analytical grade (Carlo ERBA). The reactions under inert atmosphere were carried out by using Schlenck techniques. HPLC water was used as solvent.

Synthesis of $N, N^{\prime}$-bis(2-phosphonoethyl)-3,4;9,10perylenediimide, (PDI-BP). The synthesis of the ligand (hereafter PDI-BP) has been carried out by following a previously reported method. ${ }^{15}$ The dark-red product, obtained as monoimidazolium salt, was transformed into the watersoluble bis-sodium salt of PDI-BP by addition of $\mathrm{NaOH}(1 \mathrm{M})$. After filtration the dark-red solution was exposed to vapors of $\mathrm{HCl}(37 \%)$ in a desiccator in order to precipitate the PDI-BP ligand as a dark-red-brown solid. The final product was washed with cooled water until neutrality and finally with pure ethanol. The product was dried under vacuum. The purity of the compound was checked by elemental analysis and UV-vis and infrared spectroscopy.

Synthesis of $\mathrm{Ni}_{2}\left[(\mathrm{PDI}-\mathrm{BP})\left(\mathrm{H}_{2} \mathrm{O}\right)_{2}\right] \cdot 3 \mathrm{H}_{2} \mathrm{O}$ (1). The $\mathrm{Ni}(\mathrm{II})$ compound has been prepared by mixing the ligand PDI-BP with $\mathrm{Ni}^{\mathrm{II}}$ chloride hexahydrate and urea in water in a $2: 1: 4.6$ Ni:PDI-BP:urea molar ratio. Chemical synthesis of compound (1) has been carried out under inert atmosphere using Schlenk techniques and typically $0.17 \mathrm{~g}$ of $\left[\mathrm{NiCl}_{2}\right] \cdot 6 \mathrm{H}_{2} \mathrm{O}(0.72 \mathrm{mmol})$, $0.22 \mathrm{~g}$ of PDI-BP $(0.36 \mathrm{mmol})$, and $0.09 \mathrm{~g}$ of urea $(1.5 \mathrm{mmol})$ have been suspended in $7 \mathrm{~mL}$ of ultrapure water in a flask equipped with a refrigerator under nitrogen gas. The resulting dark red reaction mixture was refluxed for two weeks under inert atmosphere. A dark-red powdered solid precipitated, which was filtered off, washed with water, acetone, and dried to the air.

Anal. Calcd for $\mathrm{C}_{28} \mathrm{H}_{26} \mathrm{~N}_{2} \mathrm{O}_{15} \mathrm{P}_{2} \mathrm{Ni}_{2}: 41.57 \% \mathrm{C} ; 3.24 \% \mathrm{H}$; $3.47 \% \mathrm{~N} ; 7.67 \% \mathrm{P}$; $14.34 \% \mathrm{Ni}$. Found: $38.51 \% \mathrm{C}$; $3.59 \% \mathrm{H}$; $3.39 \% \mathrm{~N} ; 5.85 \% \mathrm{P} ; 13.28 \% \mathrm{Ni}$.

Characterization and Physical Measurements. Elemental analyses have been performed by the Servizio di Microanalisi della Area di Ricerca di Roma del CNR and by Malissa \& Reuter, Lindlar, Germany. Thermogravimetric (TGA) data were obtained in flowing dry nitrogen at a heating rate of 10 ${ }^{\circ} \mathrm{C} / \mathrm{min}$ on a Thermoanalysis Q60 thermoanalyzer. The FTIR absorption spectra were recorded on a Shimadzu Prestige 21 spectrophotometer using $\mathrm{KBr}$ pellets. The UV-vis reflectance spectra of the powder samples were recorded on a PerkinElmer 950 spectrophotometer. Static magnetization measurements were performed with a Quantum Design MPMS-XL-5 SQUID magnetometer in the 2 to $300 \mathrm{~K}$ temperature range at different applied fields and in zero-field-cooled (ZFC) and field-cooled (FC) modes. The isothermal magnetization measurements were done with fields from -5 to $5 \mathrm{~T}$. AC susceptibility measurements were performed on the same samples with an oscillating magnetic field of $0.395 \mathrm{mT}$ in the frequency range $1-1000 \mathrm{~Hz}$. Susceptibility data were corrected for the sample holder and for the diamagnetic contribution of the salt using Pascal's constants. ${ }^{29}$

Trasmission Electron Microscopy-TEM. Trasmission Electron Microscopy observations were performed using a JEM-2100 (JEOL, Japan) operating at $100 \mathrm{kV}$ accelerating voltage, equipped with an energy dispersive $\mathrm{X}$-ray spectrometer (EDS, Oxford Instruments, UK) suited for element identification. A small quantity of $\mathrm{Ni}_{2}\left[(\mathrm{PDI}-\mathrm{BP})\left(\mathrm{H}_{2} \mathrm{O}\right)_{2}\right] \cdot 3 \mathrm{H}_{2} \mathrm{O}$ was ultrasonically dispersed in $\mathrm{EtOH}$ and a droplet of the suspension was deposited onto an holey carbon film on 300 mesh copper grid.

Angular Dispersive X-ray Data Collection. Room temperature X-ray powder diffraction data were recorded on a Seifert XRD-3000 diffractometer, Bragg-Brentano geometry, equipped with a curved graphite monochromator $\left[\lambda\left(\mathrm{CuK}_{\alpha 1,2}\right)\right.$ $=1.54056 / 1.5444 \AA]$ and a scintillation detector. The data were collected with a step size of $0.02^{\circ}, \theta-2 \theta$ and at count time of $8 \mathrm{~s}$ per step over the range $3^{\circ}<2 \theta<80^{\circ}$. The diffractometer zero point was determined from an external $\mathrm{Si}$ standard.

Energy Dispersive X-ray Diffraction - EDXD. The characterization of compounds was carried out by energy dispersive X-ray diffraction technique. The very finely pulverized sample was compressed into pellets. Data were recorded with a custom built X-ray energy scanning diffractometer, consisting of a Seifert X-ray $\mathrm{HV}$ generator supplying a water-cooled tungsten X-ray source, with maximum power of $3.0 \mathrm{~kW}$. The bremsstrahlung (braking radiation) of the X-ray source was used. Operating conditions were: high voltage supplies $50 \mathrm{kV}$, current intensity $40 \mathrm{~mA}$. A germanium solid-state detector (SSD) was used for recording the diffraction spectra. The SSD was linked to a multichannel analyzer by an electronic chain. A set of collimating slits in front of and behind the sample, two step motors for moving arms on which the source and detector were mounted, and an adjustable sample holder placed in the optical center of the diffractometer completed the set up. The complete experimental scattering parameter range, $q=0.5-180.00 \mathrm{~nm}^{-1}$ was explored by performing several measurements in correspondence with a set of scattering angles $\theta$ at $24.0^{\circ}, 15.5^{\circ}, 8.0^{\circ}, 3.0^{\circ}, 2.0^{\circ}, 1.5^{\circ} 1.0^{\circ}$, 
a)

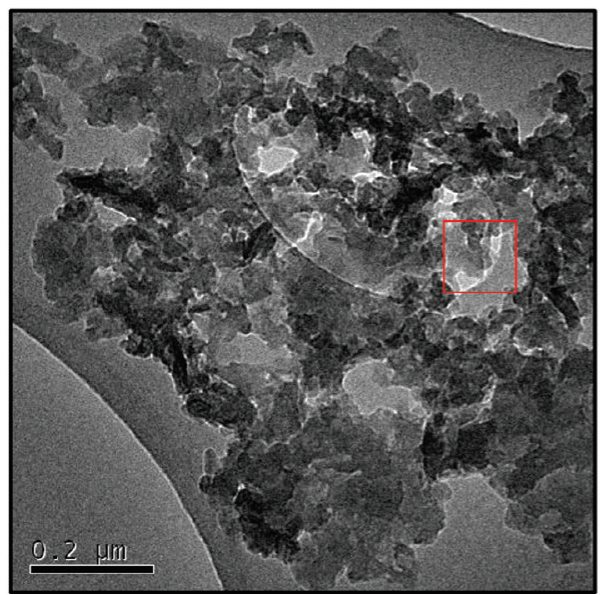

b)

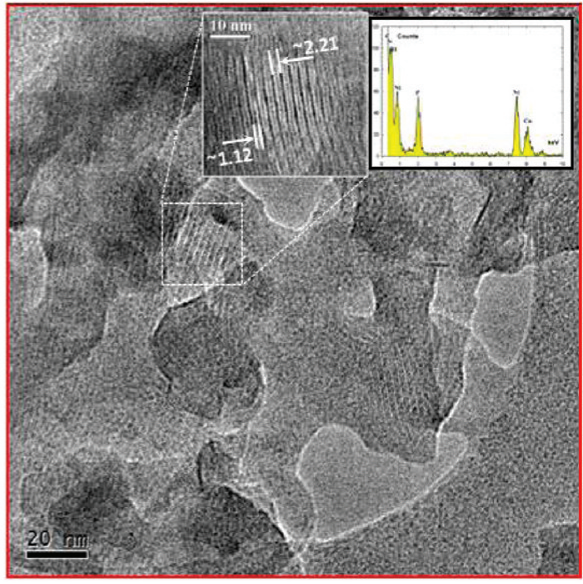

Figure 1. Evidence of self-assembled $\mathrm{Ni}_{2}\left[(\mathrm{PDI}-\mathrm{BP})\left(\mathrm{H}_{2} \mathrm{O}\right)_{2}\right] \cdot 3 \mathrm{H}_{2} \mathrm{O}$ from TEM. (a) Bright-field TEM image, (b) high-magnification image of red box shown in a. Insert: high-resolution of white box within two measured different interplanar spacing and EDS analysis probed in the same area, respectively.

$0.5^{\circ}$ and using the relation: $q=4 \pi \sin \theta / \lambda=\mathrm{EC} \sin \theta$, where $q$ is expressed in $\left[\mathrm{nm}^{-1}\right], \lambda$ in $[\mathrm{nm}]$; the used energy $(E)$ range is $13.5-38.2 \mathrm{keV}$, and the value of the constant $\mathrm{C}$ is 10.14 $\mathrm{keV} \cdot \mathrm{nm}^{-1}$. The experimental data were corrected for the following effects: escape peak suppression, normalization to the incident radiation intensity, division by X-ray absorption and polarization coefficients, and subtraction of the contributions, due to inelastic scattering, from the observed intensities. Then the spectra were joined to reconstruct the whole diffraction pattern. ${ }^{17}$

\section{RESULTS AND DISCUSSION}

$\mathrm{Ni}_{2}\left[(\mathrm{PDI}-\mathrm{BP})\left(\mathrm{H}_{2} \mathrm{O}\right)_{2}\right] \cdot 3 \mathrm{H}_{2} \mathrm{O}$ was isolated as a poorly crystalline red powder (Figure S1 of the Supporting Information) and characterized by elemental analysis and several techniques, such as TGA-DSC, TEM, EDXD as well as various spectroscopies (UV-vis, FTIR). TGA-DSC results are in agreement with the presence of five water molecules per formula unit: the loss at $\sim 100{ }^{\circ} \mathrm{C}$ can be ascribed to three crystallization water molecules and the loss in the temperature range $180-213{ }^{\circ} \mathrm{C}$ to two coordinated water molecules (Figure S2 of the Supporting Information). The conventional X-ray diffraction pattern of (1) reported Figure S1 of the Supporting Information exhibits broad peaks over it in the low $2 \theta$ angle region. However, some insights of the molecular structure of the title compound are provided from transmission electron microscopy (TEM), UV-vis, and FTIR spectroscopies. Finally, the structure was solved by energy dispersive X-ray diffraction (EDXD) technique. ${ }^{17}$

Morphology. The morphology of compound (1) was characterized by TEM (Figure 1). Low-magnification bright field images reveal the presence of microaggregates without clearly distinguishable shape (part a of Figure 1). High magnification of the specimen shows the presence of finely dispersed plate-like shaped nanocrystalline domains of approximately $25 \mathrm{~nm}$ in size randomly oriented (part b of Figure 1). High-resolution image observation exhibits lattice with different morphology and dimensions (insert).

The measured interplanar distances corresponds to $d_{\text {Org-Org }}=$ $1.12 \mathrm{~nm}$ and $d_{\text {In-In }}=2.21 \mathrm{~nm}$. This provides direct evidence of the formation of self-assembled inorganic-organic layers in agreement with the structural model based on the EDXD experimental data. The distance between two nitrogen atoms at the edges of the perylenediimide moiety is consistent with $d_{\text {Org-Org }}$ and the distance between nickel chains of two opposite sides of the perylenediimide bridge is consistent with the value of $d_{\text {In-In }}$. Energy dispersive X-ray spectroscopy (EDS) also confirms that these regions correspond to well formed alternating layers of compound (1) as shown by the high $\mathrm{Ni}$, $\mathrm{P}, \mathrm{O}, \mathrm{N}$, and $\mathrm{C}$ content detected in this areas (insert). Further information on the data analysis procedure is given in the Supporting Information.

Chemical-Physical Characterization. The free PDI-BP ligand and $\mathrm{Ni}_{2}\left[(\mathrm{PDI}-\mathrm{BP})\left(\mathrm{H}_{2} \mathrm{O}\right)_{2}\right] \cdot 3 \mathrm{H}_{2} \mathrm{O}$ were characterized by FTIR spectroscopy (Figure S3 of the Supporting Information). The free ligand shows two broad bands centered at 2760 and $2300 \mathrm{~cm}^{-1}$, which can be assigned to $\mathrm{OH}$ stretching vibrations of the $\mathrm{P}-\mathrm{OH}$ groups: these bands disappear when the metal complex is formed suggesting the complete deprotonation of the ligand. The most prominent bands of free ligand are below $1800 \mathrm{~cm}^{-1}$ and the assignments of molecular vibrations were carried out by comparison with those of parent perylenediimide $^{18}$ and phosphonate moieties. ${ }^{16}$ The strong infrared bands at 1695 and at $1650 \mathrm{~cm}^{-1}$ are characteristic for the stretching vibrations of highly conjugated carbonyl bonds of the imide group. Other prominent bands of the ligand are observed at $1594,1578,1462,1380$, and $1345 \mathrm{~cm}^{-1}$. The peaks at 1594 and $1578 \mathrm{~cm}^{-1}$ can be assigned to the perylene $\mathrm{C}=\mathrm{C}$ stretching vibration. The bands at 1345 and $1380 \mathrm{~cm}^{-1}$ are $\mathrm{C}=\mathrm{N}$ stretching modes. The $\mathrm{C}-\mathrm{H}$ wagging vibrations of the perylene ring are found at $738 \mathrm{~cm}^{-1}$ and at $810 \mathrm{~cm}^{-1}$. The solid $\mathrm{Ni}_{2}\left[(\right.$ PDI-BP $\left.)\left(\mathrm{H}_{2} \mathrm{O}\right)_{2}\right] \cdot 3 \mathrm{H}_{2} \mathrm{O}$ features in the $3700-3200 \mathrm{~cm}^{-1}$ region a very broad and intense band centered at $3450 \mathrm{~cm}^{-1}$, which could be assigned to the $\mathrm{O}-\mathrm{H}$ stretching vibrations of coordination and crystallization water molecules. The medium band at $1650 \mathrm{~cm}^{-1}$ is assigned to the $\mathrm{H}_{2} \mathrm{O}$ bending frequency. These results are in agreement with TGA studies, which suggest the coordination of two water molecules to the metal ions in the compound. Moreover, two strong and broad bands are found in the region $1100-970 \mathrm{~cm}^{-1}$ and they can be assigned to the symmetric and asymmetric $\left[\mathrm{PO}_{3}\right]^{2-}$ group stretching modes. The other vibrations observed in the free ligand (above) are also present in the $\mathrm{Ni}^{\mathrm{II}}$ derivative, confirming the presence of the ligand in the red solid.

The absorption UV-vis spectrum of pure PDI-BP ligand in water shows four main bands at $\sim 320 \mathrm{~nm}(\mathrm{~m}), 481 \mathrm{~nm}(\mathrm{~s}), 500$ 
a)

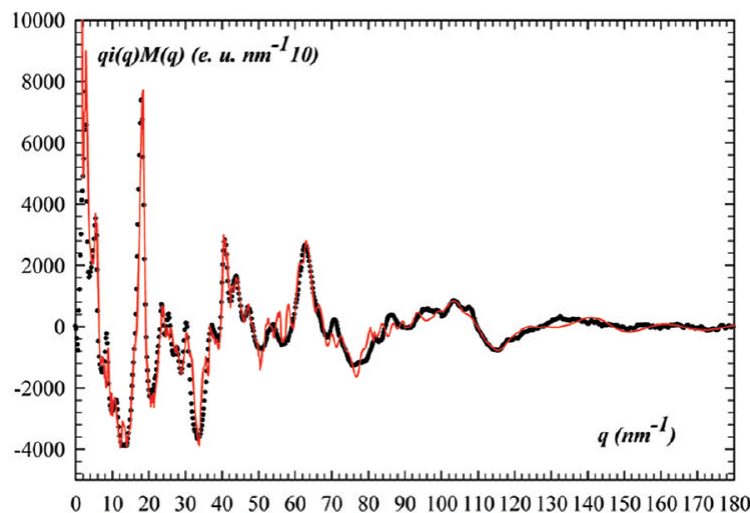

c)

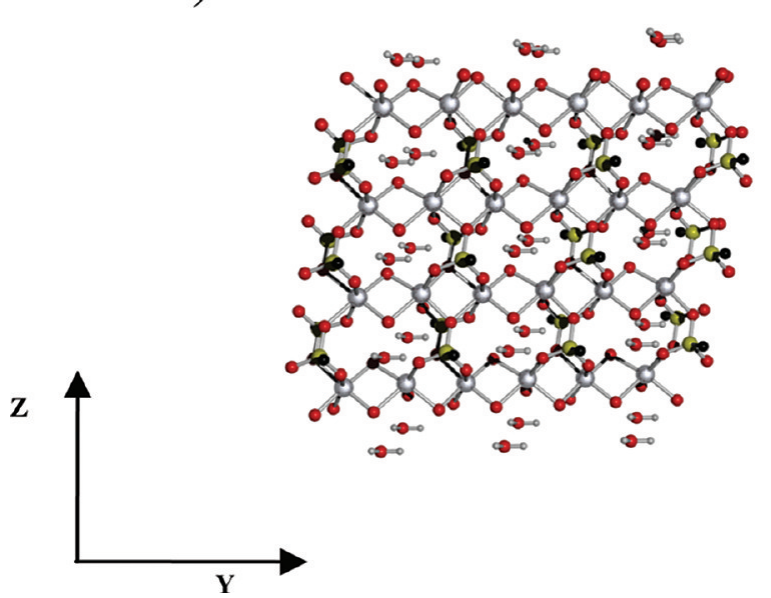

b)
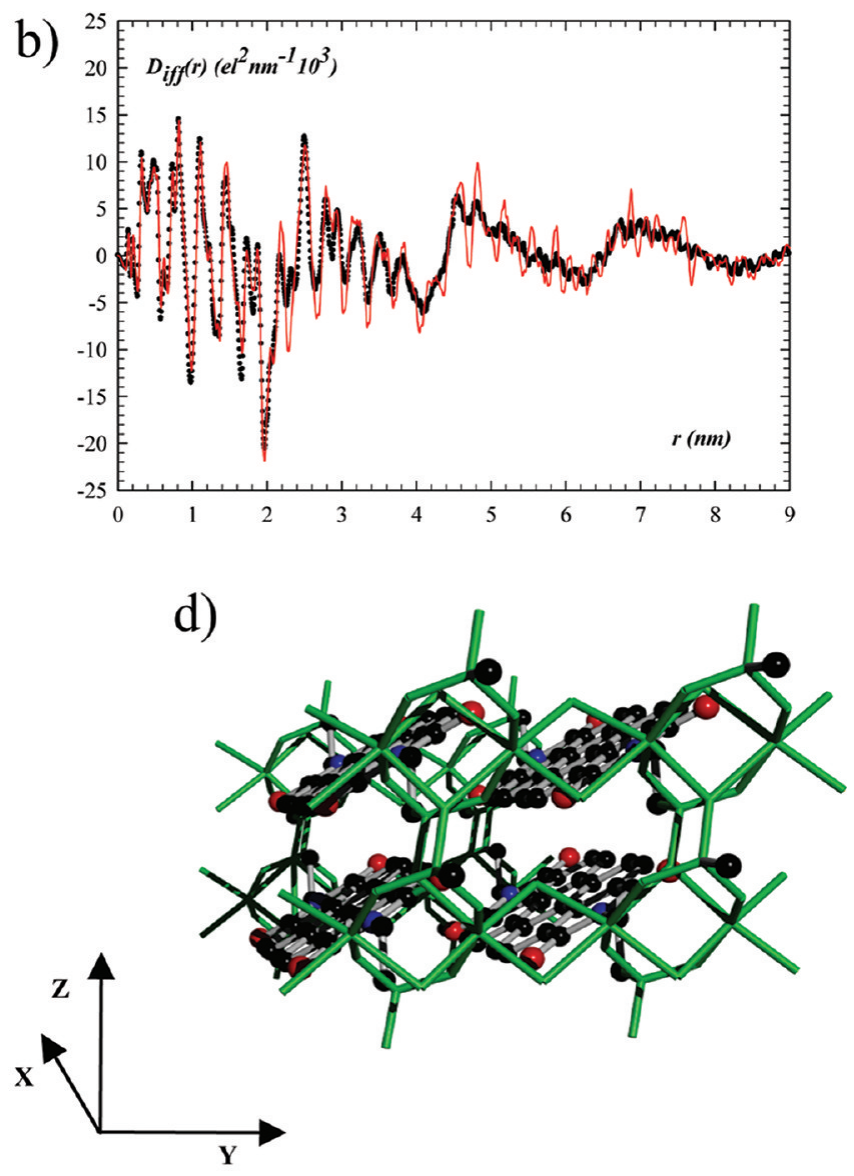

Figure 2. (Top) EDXD experiments of $\mathrm{Ni}_{2}\left[(\mathrm{PDI}-\mathrm{BP})\left(\mathrm{H}_{2} \mathrm{O}\right)_{2}\right] \cdot 3 \mathrm{H}_{2} \mathrm{O}$. a) Experimental (black points) and calculated (red-solid line) structural function $(\mathrm{SF})$ of $\mathrm{Ni}_{2}\left[(\mathrm{PDI}-\mathrm{BP})\left(\mathrm{H}_{2} \mathrm{O}\right)_{2}\right] \cdot 3 \mathrm{H}_{2} \mathrm{O}$ obtained with EDXD; b) the corresponding experimental (black points) and calculated (red solid line) Diff $(r)$. (Bottom) Two sketches in two different orientations of the structural model of $\mathrm{Ni}_{2}\left[(\mathrm{PDI}-\mathrm{BP})\left(\mathrm{H}_{2} \mathrm{O}\right)_{2}\right] \cdot 3 \mathrm{H}_{2} \mathrm{O}$ obtained by EDXD analysis. c) Inorganic layer with the following color code: $\mathrm{C}=$ black, $\mathrm{O}=$ red, $\mathrm{P}=$ yellow, $\mathrm{Ni}=$ light gray, $\mathrm{N}=$ blue; $\mathrm{d}) 3 \mathrm{D}$ view of stacked parallel organic perylene layers covalently bonded to the inorganic layer (displayed as green tube).

$\mathrm{nm}(\mathrm{s})$, and at $544 \mathrm{~nm}(\mathrm{~m})$ in agreement with the reported spectrum. ${ }^{15,19}$ The reflectance spectrum of solid $\mathrm{Ni}_{2}$ [(PDI$\left.\mathrm{BP})\left(\mathrm{H}_{2} \mathrm{O}\right)_{2}\right] \cdot 3 \mathrm{H}_{2} \mathrm{O}$ recorded in the visible and in the nearinfrared regions show five absorptions, at $\sim 335 \mathrm{~nm}(\mathrm{~m}), 380$ $\mathrm{nm}(\mathrm{w}), 503 \mathrm{~nm}(\mathrm{~s}), 550 \mathrm{~nm}(\mathrm{~s})$, and $731 \mathrm{~nm}(\mathrm{w})$. In the 1000-2000 $\mathrm{nm}$ region no bands are observed (Figure S4 of the Supporting Information).

EDXD Data Analysis and Structural Modeling. Shortmedium range structural arrangement was determined by EDXD. The experimental and calculated structure functions are reported in part a of Figure 2. The experimental structure function (SF) $q I(q) M(q)$ was collected in the reciprocal space range $0.5<q<180 \mathrm{~nm}^{-1}$ and shows the typical damped oscillations of a poorly crystalline material. The SF exhibits a high and broad peak at $2.44 \mathrm{~nm}^{-1}$ with several shoulders from 5 to $12 \mathrm{~nm}^{-1}$, a well-defined broad peak at $18.0 \mathrm{~nm}^{-1}$, and sequential overlapping peaks from 20 to $50 \mathrm{~nm}^{-1}$. These data indicate a regular structural repetition of long distances into real space. $^{17}$

At medium-high range of the SF, it is possible to observe a broad peak at $62.6 \mathrm{~nm}^{-1}$ followed by large dumping oscillations at high $q$ values, related to the inter- and intramolecular contacts. The corresponding Fourier transform, i.e. the radial distribution function (reported in the $\operatorname{Diff}(r)$ form in part $b$ of
Figure 2 in the range $0-9 \mathrm{~nm}$ ) provides direct information in real space of the inter- and intralayers contacts.

Three regions can be singled out in the $\operatorname{Diff}(r)$ function: (1) from 0.1 to ca. $0.4 \mathrm{~nm}$, mainly containing intra-inorganic contributions, (2) from ca. 0.5 to ca. $2.0 \mathrm{~nm}$, where both intrainorganic and intra-organic interactions are present, and (3) from ca. 2.0 to ca. $4.0 \mathrm{~nm}$, where the interactions between two parallel inorganic layers provide most of the contribution to the $\mathrm{X}$-ray scattering. Beyond $4.0 \mathrm{~nm}$ the intensity of the peaks becomes very small suggesting the absence, in this region, of conformational variations of the repeating units.

The structure yielding the experimental $\operatorname{Diff}(r)$ was determined by making hypothesis on the overall structure and calculating the corresponding theoretical Diff $(r)$. Subsequent refinements allowed a good agreement between theoretical and experimental curves. The starting set up for the structural determination took into account several models, other $\mathrm{Ni}$ bis-phosphonates with similar formulas ${ }^{20,21}$ and information on the organic structure gathered from previous crystallographic studies. $^{8 \mathrm{a}}$

By synchronizing all the structural parameters we determined the self-assembling structure of the $\mathrm{Ni}_{2}$ [(PDI-BP)$\left.\left(\mathrm{H}_{2} \mathrm{O}\right)_{2}\right] \cdot 3 \mathrm{H}_{2} \mathrm{O}$ platelet. In the final model, the perylene layers are stacked and shifted in the direction perpendicular to the 
stacking, in such a way that only the outer phenyl rings of the perylene planes overlap (parts $c$ and $d$ of Figure 2). The imide termination at each side is bonded to a phosphonate group. The best agreement was obtained when the perylene layers showed two slightly different distances along the stacking direction (chosen as $z$ axis) $(0.47 \pm 0.02) \mathrm{nm}$, whereas the shift of the layers with respect to each other in the perylene layer plane is $(0.15 \pm 0.03) \mathrm{nm}$ along the $x$ axis and $(0.041 \pm 0.015)$ $\mathrm{nm}$ along the $y$ direction (parts $\mathrm{c}$ and $\mathrm{d}$ of Figure 2). Furthermore, the conditions of the best fit were accomplished by using a nano-object of ten layers in stacking configuration along the $z$ axis. Each layer contains three organic-inorganic units along the $x$ axis with the organic spacer in trans configuration and 10 molecular units along the $y$ axis $(3 \times 10 \times$ 10 molecular units). The inorganic Ni chains of neighboring layers are $2.28 \mathrm{~nm}$ apart and are covalently linked by a perylene spacer in trans configuration. These values are in agreement with the lattice spacing found with TEM image analysis.

In this arrangement, the $\mathrm{Ni}^{\mathrm{II}}$ ions have an octahedral $\left[\mathrm{NiO}_{6}\right]$ coordination formed by six oxygen atoms from different shared phosponate groups. Two consecutive $\mathrm{Ni}$ atoms and two oxygen atoms are nearly planar (the dihedral angle is $5-7^{\circ}$ ) and almost perpendicular to the perylene planes. The $\mathrm{Ni}-\mathrm{O}$ bond distances range from 0.199 to $0.215 \mathrm{~nm}$. The inner octahedral angles are larger than $90^{\circ}$ (from $93.3^{\circ}$ to $98.2^{\circ}$ ). The closest $\mathrm{Ni}-\mathrm{Ni}$ distance is $(0.313 \pm 0.001) \mathrm{nm}$.

Upon fitting of the experimental data, when building up the theoretical model, we obtained the best fit with four different sets of Ni coordinates: two sets for two consecutive $\mathrm{Ni}$ atoms of the same row on one side of the perylene planes and two sets for $\mathrm{Ni}$ atoms on the other side. The calculated $\mathrm{Ni}-\mathrm{O}$ bond lengths and angles within the $\mathrm{Ni}-\mathrm{O}$ plane are reported in Tables 1 and 2 .

Table 1. Ni-O Bond Distances (in $\mathrm{nm}$ ); the Numbering of the Oxygen Atoms Is Sequential within Each Unit

$\begin{array}{lcccc} & \begin{array}{c}\mathrm{Ni}(1)^{a} \text { upper } \\ \text { unit }\end{array} & \begin{array}{c}\mathrm{Ni}(2)^{a} \text { upper } \\ \text { unit }\end{array} & \begin{array}{c}\mathrm{Ni}(1) \text { lower } \\ \text { unit }\end{array} & \begin{array}{c}\mathrm{Ni}(2) \text { lower } \\ \text { unit }\end{array} \\ \mathrm{Ni}-\mathrm{O}_{\mathrm{W}}(1)^{b} & 0.204 & 0.217 & 0.212 & 0.213 \\ \mathrm{Ni}-\mathrm{O}_{\mathrm{W}}(2) & 0.204 & 0.209 & 0.209 & 0.208 \\ \mathrm{Ni}-\mathrm{O}_{\mathrm{Ph}}(3)^{c} & 0.215 & 0.201 & 0.208 & 0.215 \\ \mathrm{Ni}-\mathrm{O}_{\mathrm{Ph}}(4) & 0.222 & 0.210 & 0.203 & 0.204 \\ \mathrm{Ni}-\mathrm{O}_{\mathrm{Ph}}(5) & 0.220 & 0.210 & 0.204 & 0.202 \\ \mathrm{Ni}-\mathrm{O}_{\mathrm{Ph}}(6) & 0.199 & 0.200 & 0.210 & 0.204\end{array}$

${ }^{a} \mathrm{Ni}(1)$ and $\mathrm{Ni}(2)$ are consecutive $\mathrm{Ni}$ atoms of the same row. ${ }^{b} \mathrm{~W}=$ water. ${ }^{c} \mathrm{Ph}=$ phosphonate ligand.

Table 2. In-Plane $\mathrm{Ni}-\mathrm{O}-\mathrm{Ni}$ Bond Angles $\left(^{\circ}\right)$ in the Four Sets of Coordinates: $\mathrm{Ni}(1)$ and $\mathrm{Ni}(2)$ in the Upper Unit, $\mathrm{Ni}(1)$ and $\mathrm{Ni}(2)$ in the Lower Unit

$\begin{array}{lcccc} & \begin{array}{c}\mathrm{Ni}(1)^{a} \\ \text { upper unit }\end{array} & \begin{array}{c}\mathrm{Ni}(2)^{a} \\ \text { upper unit }\end{array} & \begin{array}{c}\mathrm{Ni}(1) \text { lower } \\ \text { unit }\end{array} & \begin{array}{c}\mathrm{Ni}(2) \text { lower } \\ \text { unit }\end{array} \\ \begin{array}{l}\mathrm{Ni}(1)-\mathrm{O}(1)- \\ \mathrm{Ni}(2)\end{array} & 95.84 & 97.23 & 97.76 & 93.14 \\ \begin{array}{l}\mathrm{Ni}(1)-\mathrm{O}(2)- \\ \mathrm{Ni}(2)\end{array} & 93.26 & 98.15 & 95.55 & 96.26 \\ \mathrm{O}(1)-\mathrm{Ni}(1)- & 81.19 & 80.35 & 84.73 & 82.87 \\ \quad \mathrm{O}(2) & & & & \\ \mathrm{O}(1)-\mathrm{Ni}(2)- & 88.65 & 83.53 & 80.08 & 86.23 \\ \mathrm{O}(2) & & & & \end{array}$

${ }^{a} \mathrm{Ni}(1)$ and $\mathrm{Ni}(2)$ are consecutive $\mathrm{Ni}$ atoms of the same row.
In summary, the structure is formed by nearly uniform chains of edge-sharing $\mathrm{Ni}^{\mathrm{II}}$ octahedra. Adjacent chains are anchored to each other by the oxygens of one phosphonate group. The whole set of coordinates, used to build up the model is reported in Table S1 of the Supporting Information.

Magnetic Properties. The temperature dependence of the molar magnetic susceptibility, $\chi_{\mathrm{m}}$, of compound (1) per formula unit (i.e., per two $\mathrm{Ni}^{\mathrm{II}}$ ions) shows the typical increase with decreasing temperature and a sharp increase at ca. $20 \mathrm{~K}$ (part a of Figure 3) that suggests the presence of a long-range ferromagnetic ordering below this temperature. At lower temperatures, the susceptibility does not show saturation, as expected for a perfect ferromagnetic ordered state, indicating the presence of a spin canting in the long-range ordered phase (i.e., the spins are not completely parallel and present a small canting angle). The thermal variation of the $\chi_{\mathrm{m}} T$ plot (insert of part a of Figure 3) exhibits a room temperature value of ca. 2.6 $\mathrm{cm}^{3} \cdot \mathrm{K} \cdot \mathrm{mol}^{-1}$ that increases when the temperature is lowered confirming the presence of ferromagnetic $\mathrm{Ni}-\mathrm{Ni}$ interactions. At ca. $19 \mathrm{~K}, \chi_{\mathrm{m}} T$ shows a maximum followed by a decrease at lower temperatures due to the smoothening of the slope in the $\chi_{\mathrm{m}}$ plot.

The Curie plot (Figure S5 of the Supporting Information) is linear above ca. $30 \mathrm{~K}$ with a Curie constant of $2.50 \mathrm{~cm}^{3} \cdot \mathrm{K} \cdot \mathrm{mol}^{-1}$ (the expected value for two independent $S=1$ ions with $g \approx$ $2.24)^{22}$ and a Weiss temperature, $\theta=+17.2 \mathrm{~K}$ that confirms the presence of ferromagnetic interactions in the title compound. To ascertain the structural information obtained from the analysis of the EDXD data, we have used a $S=1$ regular ferromagnetic $1 \mathrm{D}$ model (eq 1$)^{23}$ to fit the magnetic data of compound (1):

$$
\begin{aligned}
\chi_{\mathrm{m}} T= & \left(2 N \beta^{2} \mathrm{~g}^{2} / 3 k_{\mathrm{B}}\right)\left[\left(A x^{3}+B x^{2}+C x+1\right)\right. \\
& \left./\left(D x^{2}+E x+1\right)\right]
\end{aligned}
$$

where $x=J / k_{\mathrm{B}} T$ and the coefficients are the following: $A=$ $0.14709, B=0.788967, C=0.866426, D=0.096573$, and $E=$ 0.624929 . This chain model reproduces very satisfactorily the magnetic data in the temperature range $25-300 \mathrm{~K}$ with $g=$ 2.259 and $J=18.6 \mathrm{~cm}^{-1}$. The positive $J$ value confirms the presence of ferromagnetic $\mathrm{Ni}-\mathrm{Ni}$ interactions along the chain. Furthermore, the fact that such a simple 1D model with only two parameters ( $g$ and $J$ ) reproduces so well the magnetic data proves the $1 \mathrm{D}$ character of the magnetic interactions in agreement with the structural results.

From the structural data, we can assume that the ferromagnetic $\mathrm{Ni}-\mathrm{Ni}$ coupling takes place through the double oxido bridges connecting the edge sharing $\left[\mathrm{NiO}_{6}\right]$ octahedra because these bridges are well known to produce a ferromagnetic coupling when the $\mathrm{Ni}-\mathrm{O}-\mathrm{Ni}$ angles are close to $90^{\circ}$. As the $\mathrm{Ni}-\mathrm{O}-\mathrm{Ni}$ angle increases, this coupling decreases and for values above ca. $97-98^{\circ}$ the coupling becomes antiferromagnetic. ${ }^{24,25}$ The moderate ferromagnetic coupling found in compound (1) suggest average $\mathrm{Ni}-\mathrm{O}-\mathrm{Ni}$ angles of ca. $95-96^{\circ}$, again in agreement with the structural data (Table 2).

The ferromagnetic ordering of compound (1) below ca. $20 \mathrm{~K}$ is confirmed by the split observed at ca. $20 \mathrm{~K}$ in the thermal variation of the zero-field cooled (ZFC) and field-cooled (FC) magnetizations (part $\mathrm{b}$ of Figure 3). Consequently, the difference between the FC and ZFC magnetizations (i.e., the irreversible magnetization, $\Delta M_{\mathrm{fc}-\mathrm{zf}}$ also shown in part b of 

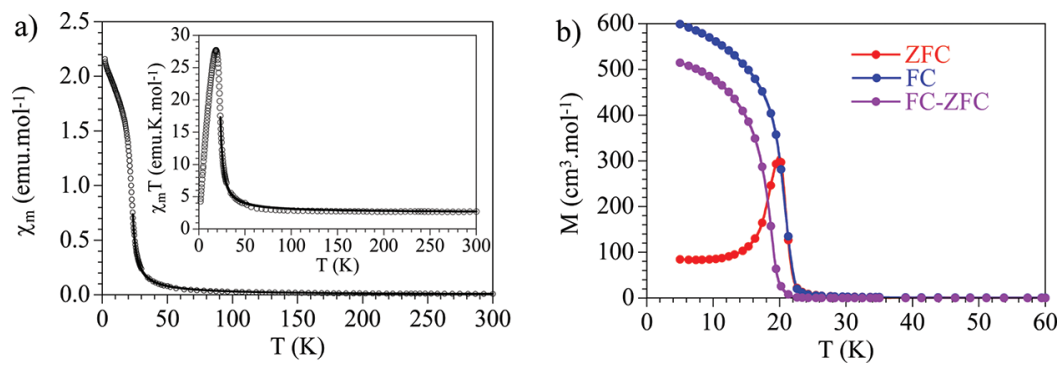

Figure 3. Magnetic properties of compound $\mathrm{Ni}_{2}\left[(\mathrm{PDI}-\mathrm{BP})\left(\mathrm{H}_{2} \mathrm{O}\right)_{2}\right] \cdot 3 \mathrm{H}_{2} \mathrm{O}$. a) Thermal variation of the molar magnetic susceptibility $\left(\chi_{\mathrm{m}}\right)$ per two $\mathrm{Ni}^{\mathrm{II}}$ ions. Insert shows the thermal variation of the $\chi_{\mathrm{m}} T$ product. Solid line is the best fit to a ferromagnetic regular $S=1$ chain model; $\mathrm{b}$ ) field cooled (blue points), zero field cooled (red points), and FC-ZFC difference (violet points) magnetizations.

Figure 3) decreases as the temperature increases and vanishes at the critical temperature $T_{\mathrm{c}} \approx 20 \mathrm{~K}$.

The existence of the ferromagnetic ordered state at low temperatures is also proven by the isothermal magnetization versus field and hysteresis measurements (the field is swept between -5 and $+5 \mathrm{~T}$ at a constant temperature in increasing and decreasing scans). These measurements show that below $20 \mathrm{~K}$ the magnetization presents hysteresis cycles (Figure 4)

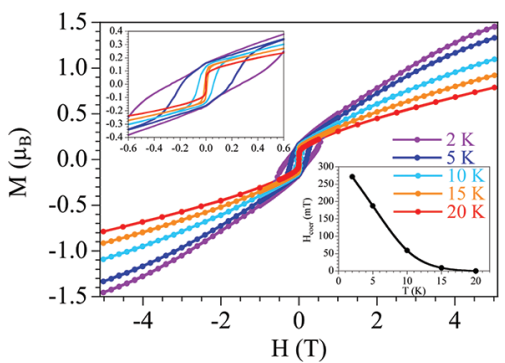

Figure 4. Hysteresis loops of the title compound per $\mathrm{Ni}^{\mathrm{II}}$ ion at 2,5 , 10,15 , and $20 \mathrm{~K}$. Left insert shows the low field region. Right insert shows the thermal evolution of the coercive field.

with coercive fields as high as $272 \mathrm{mT}(2720 \mathrm{Oe})$ at $2 \mathrm{~K}$ that decrease when the temperature is increased and vanish at $20 \mathrm{~K}$ (inserts in Figure 4). The isothermal magnetization plots also provide an additional proof of the spin canting suggested by the $\chi_{\mathrm{m}}$ versus $\mathrm{T}$ plot. Thus, the magnetization does not reach saturation even at fields as high as $5 \mathrm{~T}$ and the magnetization value at $5 \mathrm{~T}$ is below the expected one $\left(g S \approx 2.25 \mu_{\mathrm{B}}\right.$ per $\mathrm{Ni}^{\mathrm{II}}$ ion) for a perfect alignment of all the $S=1$ spins.

If we look at the first field sweep when it is increased from 0 to $5 \mathrm{~T}$, we can observe that, for $T<20 \mathrm{~K}$, the $\mathrm{M}$ versus $\mathrm{H}$ plot shows a sigmoidal shape (part a of Figure 5) with a maximumslope field $\left(H_{\mathcal{c}}\right.$ determined as the maximum in the derivative plot as seen in Figure S6 of the Supporting Information) that decreases as the temperature increases and vanishes at ca. $20 \mathrm{~K}$ (insert in part a of Figure 5). This behavior suggests the presence of a large anisotropy of the magnetic moments along the $\mathrm{Ni}$ (II) chain. The initial linear increase of the magnetization at low magnetic fields corresponds to the domain wall motion along the chain below the ordering temperature. At higher magnetic fields, there is a moment reversal that originates the rapid increase observed in the magnetic moment. As expected, the field needed to induce this moment reversal decreases as the thermal energy of the spin carriers increases (i.e., as the temperature is increased). Finally, the linear increase of the magnetization at higher fields is due to the progressive alignment of the spins in the canted ferromagnetic state.
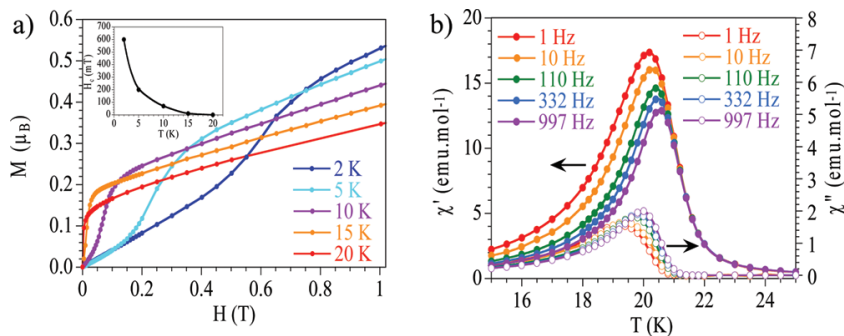

Figure 5. a) Isothermal magnetization of the title compound per $\mathrm{Ni}^{\mathrm{II}}$ ion at $2,5,10,15$, and $20 \mathrm{~K}$ in the $0-1 \mathrm{~T}$ range. Insert shows the thermal evolution of the critical field; b) ac magnetic susceptibility measurements for compound (1) at 1, 10, 110, 332, and $997 \mathrm{~Hz}$. $\left(\chi^{\prime}\right.$ filled symbols, left scale; $\chi^{\prime \prime}$ empty symbols, right scale).

Note that at $5 \mathrm{~T}$ the magnetic moment has not reached saturation (Figure 4) and the observed value (ca. 1.5 $\mu_{\mathrm{B}}$ ) is below the expected saturation value (ca. $2.2 \mu_{\mathrm{B}}$ ).

Finally, the presence of the long-range ferromagnetic ordering was ascertained by ac susceptibility magnetic measurements (i.e., with a weak oscillating magnetic field). These measurements show, as expected, a peak at ca. $20 \mathrm{~K}$ in both the in phase $\left(\chi_{\mathrm{m}}{ }^{\prime}\right)$ and the out of phase $\left(\chi_{\mathrm{m}}{ }^{\prime \prime}\right)$ susceptibilities (part $\mathrm{b}$ of Figure 5) confirming the presence of a long-range order with $T_{\mathrm{c}}=21.0 \mathrm{~K}$ (measured as the temperature where $\chi_{m}$ " becomes nonzero). A close look at both peaks show that their positions are slightly frequency dependent with an increase in the temperature of both peaks as the frequency increases from 1 to $997 \mathrm{~Hz}$. This frequency dependence is observed in spin glasses and in superparamagnetic systems (as the single molecule magnets). ${ }^{26}$ In compound (1), the frequency shift, measured as the normalized variation in the position of the peak per decade of frequency, $\Delta T_{\max } / T_{\max } \Delta(\log \nu)$, is 0.060 . This value is bigger than the normal values observed in canonical spin glasses (0.005-0.018) and is smaller than the typical values observed in superparamagnets (ca. 0.30 ) but lies within the normal range observed for spin glasslike materials $(0.06-0.08) .{ }^{26}$ Therefore, we can attribute the slight frequency dependence to the presence of some structural disorder (most probably $\mathrm{Ni}^{\mathrm{II}}$ vacancies compensated with the corresponding vacancies in the organic layers, in agreement with the chemical analysis) in the ferromagnetic layers that confers compound (1) a spin glasslike behavior in the ordered phase. This assumption is in agreement with the low crystallinity observed in the X-ray powder diffraction pattern (Figure $\mathrm{S} 1$ of the Supporting Information) and with the simultaneous presence of both ferro- and antiferromagnetic interactions within the inorganic layer. 
A further proof of the spin glasslike behavior is provided by the physically meaningless activation energy and $\nu_{0}$ values obtained from the frequency dependence of the $\chi_{m}{ }^{\prime}$ peak in the Arrhenius plot ( $\ln \nu$ vs $1 / T$, Figure S7 of the Supporting Information), in contrast to those obtained for superparamagnets $^{27}$ and from the domain wall movement phenomena in ordered systems. ${ }^{28}$

\section{CONCLUSIONS}

A new magnetic metal(II) bis-phosphonate has been prepared by chemical assembling of a relevant aromatic organophosphonate ligand with paramagnetic nickel(II) ions. The poorly crystalline red compound has been studied by EDXD in an attempt to get an insight in the structure. The fitting procedure of the resulting radial function showed a stacked layered structure, covalently bonded to the inorganic phase, with perylene planes parallel to each other and shifted in a direction perpendicular to the stacking axes, such that only the outer rings overlap. The edges of the perylene planes bind to the phosphonate groups which coordinate the nickel ions. Two bonded water molecules ensure an overall distorted octahedral $\mathrm{Ni}$ coordination. The $\mathrm{Ni}-\mathrm{O}-\mathrm{Ni}$ angles are in the range where ferromagnetic exchange interactions are expected and, accordingly, compound (1) presents a ferromagnetic intrachain coupling and weak antiferromagnetic interchain coupling through phosphonate groups. Below ca. $20 \mathrm{~K}$, compound (1) presents a long-range ferromagnetic ordering with a small canting angle and with a spin glass-like behavior due to some disorder in the inorganic layer. Furthermore, compound (1) presents hysteresis cycles with a coercive field of $272 \mathrm{mT}$ at 2 $\mathrm{K}$. This ferromagnetic ordering has been previously observed only in two $\mathrm{Ni}^{\mathrm{II}}$ organophosphonates: $\left[\mathrm{Ni}\left(\mathrm{RPO}_{3}\right)\left(\mathrm{H}_{2} \mathrm{O}\right)\right]{ }^{20} \mathrm{R}$ $=\mathrm{C}_{18} \mathrm{H}_{37}, T_{\mathrm{c}}=20 \mathrm{~K}$, and $\mathrm{Ni}_{4}\left[\left(\mathrm{O}_{3} \mathrm{P}\left(\mathrm{CH}_{2}\right) \mathrm{PO}_{3}\right)_{2}\left(\mathrm{H}_{2} \mathrm{O}\right)_{2}\right]^{21}\left(T_{\mathrm{c}}\right.$ $=4.4 \mathrm{~K})$. Compound $(1)$ demonstrates that it is possible to synthesize metal-organic ferromagnetic dyes in a quite simple way by self-assembling of a transition metal ion and an organic bis-phosphonate ligand with the appropriate donor groups.

\section{ASSOCIATED CONTENT}

\section{S Supporting Information}

Further information on EDXD data analysis, X-ray powder diffraction pattern, TGA-DSC measurement, FTIR and UVvis-NIR spectra, additional magnetic data and full list of atomic coordinates used to build up the compound model. This material is available free of charge via the Internet at http:// pubs.acs.org.

\section{AUTHOR INFORMATION}

\section{Corresponding Author}

*E-mail: elvira.bauer@ism.cnr.it (E.M.B.), roberto.matassa@ uniroma1.it (R.M.).

\section{Author Contributions}

The manuscript was written through contributions of all authors. All authors have given approval to the final version of the manuscript.

Notes

The authors declare no competing financial interest.

\section{ACKNOWLEDGMENTS}

This work is supported by Consiglio Nazionale delle Ricerche (Italy), the European Union (COST Action D35-WG11), the Spanish Ministerio de Economía e Competividad (Projects
Consolider-Ingenio 2010CSD2007-00010 and CTQ201126507), and the Generalitat Valenciana (Project PROMETEO 2009/095). We also thank Dr. P. Imperatori for helpful discussions and for the XRPD data collection. TEM experimental data were provided by Centro Grandi Apparecchiature - UniNetLab - Università di Palermo funded by POR Sicilia 2000-2006, Misura 3.15 Quota Regionale. CASPUR (Consorzio interuniversitario per le Applicazioni di Supercalcolo Per l'Università e la Ricerca) is acknowledged for providing computing facilities for EDXD data analysis.

\section{REFERENCES}

(1) (a) Jain, P.; Ramachandran, V.; Clark, R. J.; Zhou, H. D.; Toby, B. H.; Dalal, N. S.; Kroto, H. W.; Cheetham, A. K. J. Am. Chem. Soc. 2009, 131, 13625-13627. (b) Xu, G. C.; Zhang, W.; Ma, X. M.; Chen, Y. H.; Zhang, L.; Cai, H. L.; Wang, Z. M.; Xiong, R. G.; Gao, S. J. Am. Chem. Soc. 2011, 133, 14948-14951.

(2) Coronado, E.; Galán-Mascarós, J. R.; Gómez-García, C. J.; Laukhin, V. Nature 2000, 408, 447-449.

(3) (a) Zhang, Y.; Liu, T.; Sato, O. J. Am. Chem. Soc. 2010, 132, 912913. (b) Kurmoo, M. Chem. Soc. Rev. 2009, 38, 1353-1379.

(4) (a) Kurmoo, M.; Graham, A. W.; Day, P.; Coles, S. J.; Hursthouse, M.; Caulfield, J. L.; Singleton, J.; Pratt, F. L.; Hayes, W.; Ducasse, L.; Guionneau, P. J. Am. Chem. Soc. 1995, 117, 1220912217. (b) Kobayashi, H.; Tomita, H.; Naito, T.; Kobayashi, A.; Sakai, F.; Watanabe, T.; Cassoux, P. J. Am. Chem. Soc. 1996, 118, 368-377. (c) Coronado, E.; Curreli, S.; Giménez-Saiz, C.; Gómez-García, C. J. J. Mater. Chem. 2005, 15, 1429-1436. (d) Coronado, E.; Curreli, S.; Giménez-Saiz, C.; Gómez-García, C. J. Inorg. Chem. 2012, 51 (2), $1111-1126$

(5) See for example: Functional Hybrid Materials; Gómez-Romero, P., Sanchez, C., Eds.; Wiley-VCH: Weinheim, Germany, 2004.

(6) (a) Clearfield, A. Prog. Inorg. Chem. 1998, 47, 371-510. (b) Bellitto, C. In Magnetism: Molecules to Materials, Vol. 2 , Miller, J. S.; Drillon, M., Eds.; Wiley-VCH: Weinheim, 2001; p 425-456; (c) Bellitto, C.; Bauer, E. M.; Righini, G. Inorg. Chim. Acta 2008, 361, 3785-3799. (d) Gagnon, K. J.; Perry, H. P.; Clearfield, A. Chem. Rev. 2012, 112, 1034-1054.

(7) Bhosale, S. V.; Jani, C. H.; Langford, S. J. Chem. Soc. Rev. 2008, 37, 331-342.

(8) (a) Würthner, F. Chem. Commun. 2004, 1564-1579. (b) Huang, C.; Barlow, S.; Marder, S. A. J. Org. Chem. 2011, 76, 2386-2407.

(9) Zollinger, H. In Color Chemistry, $3^{\text {rd }}$ Edn., Wiley-VCH: Weinheim, 2003.

(10) Shirman, E.; Ustinov, A.; Ben-Shihit, N.; Weissman, H.; Iron, M. A.; Cohen, R.; Rybttchinski, B. J. Phys. Chem. B 2008, 112, 88558858.

(11) (a) Newmann, C. D.; Friesbe, C. D.; Silva-Filho, D. A.; Bredas, J. L.; Ewbank, P. C.; Mann, K. R. Chem. Mater. 2004, 16, 4436-4451.

(b) Dimitrakopoulos, C. D.; Malefant, P. R. L. Adv. Mater. 2002, 14, 99-117.

(12) Gregg, B. A.; Cormier, V. J. Am. Chem. Soc. 2001, 123, 79597960.

(13) Struijk, C. W.; Sieval, A. B.; Dakhorst, J. E. J.; VanDijk, M.; Kinkes, P.; Koehorst, R. B. M.; Schaafsma, T. J.; Picken, S. J.; van de Craats, A. M.; Warman, J. M.; Zuilhof, H.; Sudhölter, E. J. R. J. Am. Chem. Soc. 2000, 122, 11057-11066.

(14) Würthner, F. Angew. Chem., Int. Ed. 2001, 40, 1037-1039.

(15) (a) Marcon, R. O.; Dos Santos, J. G.; Figueiredo, K. M.; Brochsztain, S. Langmuir 2006, 22, 1680-1687. (b) Marcon, R. O.; Brochsztain, S. Thin Solid Films 2005, 492, 30-34.

(16) Bellitto, C.; Bauer, E. M.; Righini, G.; Gómez-García, C. J. J. Solid State Chem. 2008, 181, 1213-1219.

(17) (a) Matassa, R.; Carbone, M.; Lauceri, R.; Purrello, R.; Caminiti, R. Adv. Mater. 2007, 19, 3961-3967. (b) Capobianchi, A.; Pennesi, G.; Paoletti, A. M.; Rossi, G.; Caminiti, R.; Sadun, C.; Ercolani, C. Inorg. Chem. 1996, 35 (16), 4643-4648. (c) Ballirano, P.; Caminiti, R. J. 
Appl. Crystallogr. 2001, 34 (6), 757-762. (d) Carbone, M.; Caminiti, R.; Sadun, C. J. Mater. Chem. 1996, 6 (10), 1709-1716.

(18) (a) Rodriguez-Llorente, S.; Arroca, R.; Duff, J. J. Mater. Chem. 1998, 8, 2175-2179. (b) Arroca, P.; Arroca, R.; Kovacs, G. J.; Loutfy, R. O. Langmuir 1990, 6, 1050-1054.

(19) Marcon, R. O.; Brochsztain, S. J. Phys. Chem. A 2009, 113, $1747-1752$.

(20) Bellitto, C.; Bauer, E. M.; Righini, G.; Portalone, G.; Drillon, M.; Rabu, P. Inorg. Chem. 2008, 47, 10945-10952.

(21) Gao, Q.; Guillou, N.; Nogues, M.; Cheetham, A. K.; Ferey, G. Chem. Mater. 1999, 11, 2937-2947.

(22) Carlin, R. L. Magnetochemistry; Springer-Verlag: Berlin, 1986.

(23) Monfort, M.; Resino, I.; Fallah, M. S. E.; Ribas, J.; Solans, V.; Font-Bardia, M.; Stoeckli-Evans, H. Chem.-Eur. J. 2001, 7, 280-287.

(24) Bertrand, J. A.; Ginsberg, A. P.; Kaplan, R. I.; Kirkwood, C. E.; Martin, R. L.; Sherwood, R. C. Inorg. Chem. 1971, 10, 240-246.

(25) (a) Halcrow, M. A.; Sun, J. S.; Huffman, J. C.; Christou, G. Inorg. Chem. 1995, 34, 4167-4177. (b) Clemente-Juan, J. M.; Coronado, E.; Galán-Mascarós, J. R.; Gómez-García, C. J. Inorg. Chem. 1999, 38, 55-63. (c) Clemente-Juan, J. M.; Chansou, B.; Donnadieu, B.; Tuchages, J. P. Inorg. Chem. 2000, 39, 5515-1519. (d) Mukherjee, P.; Drew, M. G. B.; Gómez-García, C. J.; Ghosh, A. Inorg. Chem. 2009, 48, 5848-5860. (e) Sadhukhan, D.; Ray, A.; Pilet, G.; Rizzoli, C.; Rosair, G. M.; Gómez-García, C. J.; Signorella, S.; Bellú, S.; Mitra, S. Inorg. Chem. 2011, 50, 8326-8339. (f) Banerjee, S.; Nandy, M.; Sen, S.; Mandal, S.; Rosair, G. M.; Slawin, A. M. Z.; Gómez García, C. J.; Clemente-Juan, J. M.; Zangrando, E.; Guidolin, N.; Mitra, S. Dalton Trans. 2011, 40, 1652-1661.

(26) Mydosh, J. A. Spin Glasses: An Experimental Introduction; Taylor \& Francis: London, 1993.

(27) (a) Sessoli, R.; Powell, A. K. Coord. Chem. Rev. 2009, 253, 2328-2341. (b) Gatteschi, D.; Sessoli, R. Angew. Chem., Int. Ed. 2003, 42, 268-297.

(28) (a) Coronado, E.; Gómez-García, C. J.; Nuez, A.; Romero, F. M.; Rusanov, E.; Stoeckli-Evans, H. Inorg. Chem. 2002, 41, 46154617. (b) Bellouard, F.; Clemente-León, M.; Coronado, E.; GalánMascarós, J. R.; Gómez-García, C. J.; Romero, F. M.; Dunbar, K. R. Eur. J. Inorg. Chem. 2002, 1603-1606. (c) Triki, S.; Sala-Pala, J.; Thetiot, F.; Gómez-García, C. J.; Daran, J. C. Eur. J. Inorg. Chem. 2006, 185-199. (d) Coronado, E.; Gómez-García, C. J.; Nuez, A.; Romero, F. M.; Waerenborgh, J. C. Chem. Mater. 2006, 18, 2670-2681.

(29) Bain, G. A.; Berry, J. F. J. Chem. Educ. 2008, 85, 532-536. 